\title{
Nurses' Perspectives on Patient and Visitor Violence: A Qualitative Study
}

\author{
Lannette Henderson ${ }^{1}$, Brittany Kamp ${ }^{2}$, Keri Niedbalski ${ }^{3}$, Samuel P. Abraham ${ }^{4}, \&$ Deborah R. Gillum ${ }^{5}$ \\ ${ }^{1}$ Psychiatric Acute Care Unit, Memorial Epworth Center, South Bend, Indiana, USA \\ ${ }^{2,3}$ Emergency Room, Elkhart General Hospital, Elkhart, Indiana, USA \\ ${ }^{4,5}$ Bethel College School of Nursing, Mishawaka, Indiana, USA \\ Correspondence: Samuel P. Abraham, Associate Professor of Nursing, Bethel College School of Nursing, 1001 \\ Bethel Circle, Mishawaka, Indiana, 46545, USA.
}

Received: July 1, 2018

Accepted: July 14, 2018

Online Published: July 24, 2018

doi:10.20849/ijsn.v3i2.427

URL: https://doi.org/10.20849/ijsn.v3i2.427

\begin{abstract}
The profession of nursing is at high risk for work-related threats and violence from patients and visitors. The purpose of this study was to investigate nurses' perspectives on patient and visitor violence. In this research study, a qualitative, phenomenological design was used. The study question was, "What are your lived experiences as a nurse with patient and visitor violence?" This study included what nurses have personally experienced and how they handled violent situations while performing their job. A total of 19 registered nurses were interviewed. Open-ended questions and follow-up probes evoked responses. Sequential interviews were conducted until all concepts were repeated multiple times without new themes emerging. Four common themes emerged were violence (punching, kicking, tackling, pinching, and spitting), long-term consequences of violence, need for education and training for violence, and the need for support from the organization. Taylor's cognitive adaptation adjustments to threatening events theory helped guide the study.
\end{abstract}

Keywords: nurses, workplace violence, psychological violence, physical violence, verbal violence, coping, stress, anxiety, depression

\section{Introduction}

Physical, verbal and emotional violence against nurses, in all areas of healthcare, is increasing worldwide. A report from the United States, Bureau of Labor Statistics (2016) indicated the rate of injury in the healthcare setting was three times greater than the rate in private industries. The American Nursing Association (ANA, 2015) released a report, which indicated that " $43 \%$ of nurses and nursing students had been verbally or physically threatened by a patient or patient's family member and $24 \%$ had been assaulted" (p. 4). The purpose of this qualitative, phenomenological study was to determine nurses' perspectives on patient and visitor violence. This was conducted with open-ended, in-depth interviews with nurses until data saturation was reached.

\section{Background}

Lived experiences by the researchers motivated this study. Working as support staff in emergency and psychiatric units, violence is a commonly seen occurrence. In first-hand experiences, violence by patients and visitors has been detrimental to the staff and workplace environment. Research the effects of violence against nurses can result in physical injury, increased stress, and anxiety. Baran-Aksakal, Karasahin, Urgas-Dikmen, Avci, and Ozkan (2014) added that nurses were three times more likely than individuals in other professions to experience violence in the workplace (p. 1366).

Reports by the Emergency Nurses Association (ENA) and Occupational Safety and Health Administration (OSHA) indicated that workplace violence (WPV) could be prevented, or the risk at least minimized, when employers take necessary precautions (as cited in Stene, Larson, Levy, \& Dohlman, 2015, p. 113). Kvas and Seljak (2014) stated that "accurate data about WPV does not exist because the majority of violent acts remain unreported. To reduce WPV, we first need to establish its prevalence and based on the data on the scope, type, and degree of violence, adopt appropriate measures for its prevention and reduction" (p. 244). It is important to make nurses' experiences and thoughts regarding patient and visitor violence known so that steps can be taken to decrease the incidence. 
Zhao et al. (2015) affirmed with the encouragement of health care organizations and the larger society; changes can be made.

Avander, Heikki, Bjersa, and Engstrom (2016) reported that the profession of nursing is at high risk of work-related threats and violence (p. 55). The World Health Organization (WHO, 2017) indicated that physical violence is experienced by $8-38 \%$ of healthcare workers, and the incidence of verbal abuse is much higher. This type of violence included direct physical assault, homicide, physical harassment, written or verbal threats, physical or verbal harassment, and homicide (OSHA, 2015). The proposed study was to explore violence experienced by nurses in their workplace in northern Indiana.

\section{Purpose and Study Question}

The purpose of this qualitative, phenomenological study was to investigate nurses' perspectives of patient and visitor violence. Two questions were the focus of this study: What are nurses' lived experiences of violence by patients? What are nurses' lived experiences of violence by visitors?

\section{Review of the Literature}

Scholarly peer-reviewed journal articles were used for this research study. Cumulative Index of Nursing and Allied Health Literature (CINAHL) and Medline Full Text were the databases used to locate the articles. The College library database was also used to search for articles using the key terms: nurses; workplace violence; psychological violence; sexual violence; physical violence; verbal violence; coping; stress; anxiety; and depression. The peer-reviewed articles were published from 2014 to 2017.

\subsection{Types of Violence}

Many types of violence occur against nurses in the healthcare setting, namely verbal, physical, and emotional. Baran-Aksakal et al. (2014) found that in a study of 538 nurses, $13.9 \%$ described experiencing physical violence, $41.8 \%$ experienced verbal violence, and $17.1 \%$ described being mugged (p. 1361). This is a significant number of nurses experiencing some type of violence in the healthcare setting. Stevenson, Jack, O'Mara, and LeGris (2015) reported that psychiatric nurses had the highest victimization rates of all types of nurses, so much that they considered violence a part of the job. Stevenson et al. revealed that $55 \%$ of psychiatric nurses were victims of verbal or emotional abuse and $19.5 \%$ were sexually abused.

Violence is not only instigated by patients, but also by their relatives and visitors. Baran-Aksakal et al. (2014) reported that $61.3 \%$ of nurses described physical violence by patients 'visitors, and that $54.7 \%$ of verbal abuse was from visitors as well (p. 1361). There is a higher incidence of visitor violence toward nurses, rather than patient violence against nurses. In addition to violence by visitors, Baran-Akasakal et al. (2014) reported $42.7 \%$ of physical violence was by patients, and that $32.6 \%$ of the verbal violence against nurses was from patients as well (p. 1361). "Verbal and physical abuse by family members toward nurses could be associated with the high-stress conditions and frustration over imbalances in authority between the nurse and the family" (Trahan \& Bishop, 2016, p. 119).

Verbal violence is one of the more common types of violence projected toward nurses. Edward, Ousey, Warelow, and Lui (2014) discovered that verbal abuse was the most frequently encountered form of violence and aggression towards nurses (p. 9). Edward et al. stated that verbal abuse from patients or their relatives towards nurses was evident in many countries, including prevalence rates in Canada of $94 \%$, Turkey of $91 \%$, and the United Kingdom had a prevalence rate over $90 \%$ (p. 10). Edward et al. described verbal abuse as yelling, being sworn at, being intimidated, and being harassed by sexual language or suggestions (p. 10).

Avander, Heikki, Bjersa, and Engstrom (2016) described the indirect verbal violence as patients or visitors using a certain tone of voice when speaking or used indirect threats towards nurses (p. 53). This was often seen when patients were not happy with their care or they were not satisfied with what was done for them. Often, patients used indirect verbal threats towards nurses when they were talking to family members or visitors about how they were unpleased with their care. Avander et al. concluded that patient violence towards nurses was more common than visitor violence toward nurses (p. 54).

Violence affects every nurse in the healthcare setting, but studies have found higher rates of violence towards male nurses and new graduate nurses. Edward et al. (2014) reported that "younger and less experienced nurses were at a higher risk for workplace violence than more experienced nurses" (p. 7). Edward et al. also explained that male nurses were more likely to experience physical violence than female nurses (p. 7).

Evidence has indicated that violence toward health care workers happens more during shifts that have fewer workers on duty. Edward et al. (2014) reported that "night shift nurses and weekend nursing staff were more at 
risk of workplace violence, and it may be attributed to the relative isolation in which these nurses are working and lower staffing levels during these 'quieter' times" (pp. 7-8).

All health care settings have occurrences of physical, verbal, or emotional violence. Edward et al. (2014) stated, "violent/physical assaults were identified as more prevalent in mental health settings, geriatrics, long-term care, and nursing homes than in general clinical environments" (p. 11). Another clinical setting that had high incidences of physical violence towards nurses was the emergency department (ED). Edward et al. found that the incidence of physical assaults towards U. S. nurses in the ED was $82 \%$ (p. 11).

Zhao et al. (2015) completed a cross-sectional survey of health care professionals from 19 hospitals in six Chinese cities between July and October 2014. Approximately, 126 participants from each of the 19 hospitals were chosen. A questionnaire developed in 2003 by the International Labor Office (ILO), International Council of Nurses (ICN), Public Services International (PSI), and WHO was used to survey the individuals. Information collected included demographics, workplace violence anxiety, lived experiences, and prevention. Of the 1,793 health care professionals who responded, $170(9.5 \%)$ had been exposed to physical violence, and 1,241 $(69.2 \%)$ had been exposed to psychological violence over the previous 12 months (Zhao et al., 2015).

\subsection{Lack of Leadership Support}

Many nurses do not report workplace violence. Some nurses do not believe that they get enough support or education on how to appropriately respond to these situations in the workplace. Edward et al. (2014) reported rates of up to $80 \%$ of non-reporting of violence in the workplace (p.13). Baran-Aksakal et al. (2014) explained that reporting does not happen because of the "inability to follow up and complete legal procedures, [a] lack of a reporting system, and unsatisfactory responses from supervisors" (p. 1366). Edward et al. mentioned similar barriers to reporting violence, including poor or no support following an incident, lack of an incident reporting system, a previous incident without action, and fear of adverse occupational consequences (p. 12).

Kvas and Sejak (2014) surveyed 692 nurses in Slovenia, and 62\% of those nurses had been exposed to at least one form of violence in the previous year. A small portion (6.5-10.9\%) reported the incident in written form. Most of the victims (51.7-77.8\%) did not report the violence because of the belief that it would not change anything or they had a negative experience reporting it in the past. As shown in Figure 1, respondents believed that education and training would have a significant impact on improving the issue (Kvas \& Sejak, 2014)

\subsection{Need for Training and Education}

Many nurses believed that violence is just 'part of the job', but however, it should not be tolerated. Stene, Larson, Levy, and Dohlman (2015) conducted a study using the Emergency Department Assessment Tool provided by the ENA. Even though $75 \%$ of the 114-member nursing care team had experienced abuse in the month before being surveyed, $67 \%$ had not reported it. One year after a concise reporting tool and an educational program was implemented, a follow-up survey of 120 individuals showed that with education, a proper reporting tool, and leadership support, a significant increase in reporting violence occurred. The number of staff who considered workplace violence to be part of their job decreased by half.

\subsection{Consequences of Violence on Overall Health}

Violence towards nurses has negative long-term effects on overall health and well-being. Baran-Aksakal et al. (2014) reported that "nurses who did not experience verbal violence at work were more satisfied with their profession, compared to those who had experienced violence reported that they were more willing to change their profession" (pp. 1361-1364). Baran-Aksakal et al. reported that "exposure to violence has adverse effects on mental health, and that experiencing violence can lead to anxiety, frustration, anger, fear, dissatisfaction with work, posttraumatic stress disorder, sleep disorders, physical harm (including death and loss of consciousness), loss of work days, loss of skills, resignation from work, changing of work, and negative effects on the provision of health services" (p. 1367).

Nurses cope and react to traumatic experiences differently. Edward et al. (2014) described that "exposure to traumatic experiences over a career of nursing, and a lack of control over these experiences, contributes to poor recruitment, poor retention, and may manifest as exhaustion" (p. 2). With the current nursing shortage, exposure to violence may contribute to difficulty with retaining nurses in health care settings.

Feelings often experienced after a traumatic event can cause individuals to have stress in their everyday life. Edward et al. (2014) added that exposure to traumatic events led to feelings of anger, being cynical and negative in the workplace, and being physically exhausted, which can lead to depression and anxiety (p. 2). Nurses have many emotions after experiencing a traumatic event. Edward et al. stated that nurses reported "experiencing sadness, shock, confusion, anger, and embarrassment following an aggressive incident" (p. 11). 
Avander et al. (2016) found the risk for violence from patients and families had an impact on the nurse-patient relationship (p. 54). Violence from a patient or their family member caused tension between the nurse and the patient. Avander et al. discovered that when patients or visitors addressed the nurses in a threatening manner, the nurses felt fear and often ignored patients or they 'gave in' to the patient or visitors demands (p. 54). Avander et al. reported that when a violent situation occurred, occasionally nurses left the room or even avoided doing nursing interventions at the appropriate time (p. 55). Nurses avoided patients because of fear of how the patient or visitors would react to the violent incident. Violence has affected the quality of nursing care that individuals receive in the healthcare setting.

Violent patients and visitors also affect nurses' lives outside of the healthcare setting. Avander et al. (2016) described when violent situations occurred in the workplace; nurses were often afraid of how it would affect their lives outside of the hospital (p. 55). Nurses were concerned about chance interactions with these patients outside of the hospital. Avander et al. found that nurses often had general feelings of insecurity, anxiety, and fear of an extreme outburst if they encountered the violent individual outside of the hospital (p. 55).

Baby, Glue, and Carlyle (2014) confirmed through semi-structured interviews of 13 registered nurses (RNs) and 1 licensed practical nurse (LPN) from a mental health unit in New Zealand that verbal abuse was experienced daily. Physical violence was experienced, but not as often as verbal violence. Negative side effects seen in nurses after these experiences included anxiety, frustration, and burnout (Baby et al., 2014).

\subsection{Summary of the Literature Review}

In summary, the literature review confirmed that nurses experience violence in the work setting. The information confirmed that nurses experience physical, verbal, and emotional abuse. Nurses also reported inadequate support from leadership to address patient and visitor violence. It was discovered that many violent activities were not reported and increased reporting would help decrease violent occurrences (see Figure 1). The literature review supports that there are long-term consequences of violence against nurses. Nurses often experience emotional problems and high rates of turnovers. The need for further research on this topic is critical.

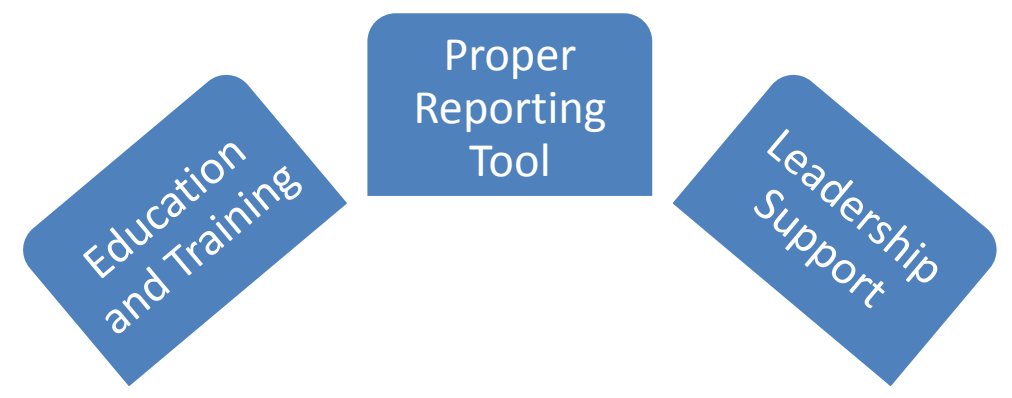

Figure 1. How to increase reporting of patient and visitor violence?

\section{Theoretical Framework}

The theoretical framework used for this study was Taylor's cognitive adaptation adjustments to threatening events theory. Taylor (1983) proposed that "the adjustment process centers around three themes: A search for meaning in the experience; an attempt to regain mastery over the event in particular and in life more generally; and an effort to restore self-esteem through self-enhancing evaluations" (p. 1161). The three themes presented in this theory were originally applied to a cancer patient's coping efforts.

The focus of this study was to determine nurses' perspectives on patient and visitor violence. The cognitive adaptation theory asserted that adapting to life events is an adjustment process focused on finding ways to feel good about oneself again (Taylor, 1983, p. 1161). The three themes helped guide the study that nurses are seeking understanding of why the event happened and what they could do differently to prevent it from happening in the future.

\section{Definition of Terms}

\subsection{Conception Definitions}

Taylor's (1983) first theme, finding meaning in the experience, is an essential step for the nurse to understand the reason that the violent event occurred. A nurse may ask: (1) what is the significance of the event? and (2) what caused the event to occur? In this example, the nurse is searching for the meaning to understand the event. The 
second theme is an attempt to regain mastery over the event. This prompts a refocusing of therapeutic communication between the nurse and the patient (Taylor, 1983). This theme centers on gaining control over the event and one's life. A nurse may ask the question, How can I keep this and similar events from happening again? The third theme is an attempt to restore self-esteem is a critical step that enables the nurse to regain confidence and functionality (Taylor, 1983).

\subsection{Operational Definitions}

Physical violence is defined as any act of beating, kicking, slapping, stabbing, shooting, pushing, biting, or pushing. Psychological violence is the threat of physical force or verbal assault against another person or group, which can result in mental, moral, physical or social development. A nurse is any individual with a registered nurse license, practicing or not practicing. Perspectives are one's own lived experiences and insights on a topic. The patient is any individual who received care from a nurse. A visitor is any individual in the healthcare setting who is not a patient or a staff member. A person is an individual. A threatening event is any event which imposed danger or fear and causes emotional or physical stress on an individual.

\section{Methodology}

\subsection{Design}

This was a qualitative, phenomenological research study to explore nurses' perspectives on patient and visitor violence. Schmidt and Brown (2015) described qualitative research as "research that uses words to describe human behaviors" (p. 543). The researchers used pre-prepared questions to interview RNs about their perspectives of patient and visitor violence. Each researcher interviewed RNs using the questions and prompts until data saturation was reached. The sample size was 19 participants. The RNs interviewed were ages 18 years and older. They were employed in any setting related to nursing. The study was conducted following approval from the College Institutional Review Board (IRB). The researchers have completed the National Institute of Health (NIH) Office of Extramural Research certification.

\subsection{Sample}

This study took place in March and April 2018. Some of the participants were known to the researchers. A snowball sampling was used to recruit additional participants. A total of 19 RNs were interviewed. Each interview took approximately 45 to 60 minutes. A researcher sat down one-on-one with each RN during the interview. The participants were instructed to answer the research question openly and honestly. There were no perceived risks for the individual to participate in this research study. The participant was given a copy of the informed consent to sign prior to the interview taking place. No names were listed on the interview documents and all answers were kept confidential.

\subsection{Setting}

The researchers interviewed each $\mathrm{RN}$ in a distraction-free location of their convenience outside of the work setting. The interviews were conducted in an area convenient to participants that was not located in the RN's work environment. The researchers ensured privacy during the interview so that all answers were kept confidential. The researchers recorded the interview and transcribed it verbatim for detailed thematic analysis. Once the recordings were transcribed, the recordings were deleted.

\section{Data Collection}

At the end of each initial interview, the researcher informed the participant of the need to follow-up after the interview had been transcribed to ensure that the identified themes reflected their experiences. After completion, the data was validated by the researchers and the professor by using qualitative content analysis.

The following steps represent Colaizzi process for phenomenological data analysis (cited in Sanders, 2003): Each transcript was read and re-read to obtain a general sense of the whole content. For each transcript, significant statements that pertain to the phenomenon under study were extracted. These statements were recorded on a separate sheet noting their pages and line numbers. Meanings were formulated from these significant statements. The formulated meanings were sorted into categories, cluster of themes, and themes. The findings of the study were integrated into an exhaustive description of the phenomenon under study. The fundamental structure of the phenomenon was described. Finally, validations of the findings were sought from the research participants to compare the researcher's descriptive results with their experiences. (pp. 22-32)

\section{Informed Consent and Confidentiality}

Privacy and confidentiality of participants were maintained. First, the College Institutional Review Board (IRB) approval was obtained prior to data collection. Secondly, the participants signed an informed consent before 
participating and were given a copy of the form. Third, the transcribed interviews were kept anonymous by excluding name and other identifying information. Informed consents and interviews were stored separately and not associated together. All collected data for this research was submitted to the College School of Nursing to be stored electronically for three years. The School of Nursing staff scanned the data into the computer and stored it on discs in a locked cabinet in a locked storage room. Only nursing administrators or the research coordinator have access to the stored records. The participants denied any distress from talking about their experience.

\section{Interview Guide}

After a thorough review of the literature, the interview guide was created to help answer the research question, "What are nurses' lived experiences of violence by a patient?" and "What are nurses' lived experiences of violence by a visitor?" Five demographic questions were also included. These questions included gender, age-range, ethnic group, degree obtained, and specialty in nursing. The interview questions were sent to two peers and two professors to review and establish face validity. Schmidt and Brown (2015) defined face validity as "a test for content validity when colleagues or subjects examine an instrument and are asked whether it appears to measure the concept" (p. 275). Although face validity was obtained, reliability could not be guaranteed.

\section{Data Analysis}

\subsection{Demographics}

The sample size included 19 participants, 17 of which were female and 2 were males (see Table 1). There were three participants ranging from age 18-25 years, seven from age 26-35, three from age 36-45, three from age 46-55, one from age 56-65, and two from age 65 and above. There were four different ethnic groups represented, Caucasian (78.9\%), African-American (10.5\%), Asian (5.3\%), and Hispanic (5.3\%). Participants held one of three degree types: Associate Degree in Nursing (26.3\%), Bachelor of Science in Nursing (68.4\%), or a Master in Nursing Degree $(5.3 \%)$. There were three different specialties represented by the 19 participants: Emergency Department (73.7\%), Psychiatric (10.5\%), and Medical-Surgical (15.8\%).

Table 1. Demographic characteristics

\begin{tabular}{lll}
\hline Variable & $\boldsymbol{f}$ & \% \\
\hline Gender & 17 & 89.5 \\
Female & 2 & 10.5 \\
Male & & \\
Age & 3 & 15.8 \\
$18-25$ & 7 & 36.8 \\
$26-35$ & 3 & 15.8 \\
$36-45$ & 3 & 15.8 \\
$46-55$ & 1 & 5.3 \\
56-65 & 2 & 10.5 \\
66+ & & \\
Ethnicity & 15 & 78.9 \\
Caucasian & 1 & 5.3 \\
Hispanic & 1 & 5.3 \\
Asian & 2 & 10.5 \\
African-American & & \\
Degree & 5 & 26.3 \\
ADN & 13 & 68.4 \\
BSN & 1 & 5.3 \\
MSN & 14 & 73.7 \\
Specialty & 2 & 10.5 \\
Emergency Room & 3 & 15.8 \\
Psychiatric & &
\end{tabular}

Note. $N=19$ 


\subsection{Themes}

Four common themes were deduced when analyzing the data from the research (see Figure 2). These themes were violence, long-term consequences of violence, education for violence, and support from the organization. The participants were assigned numbers 1-19 for this study.

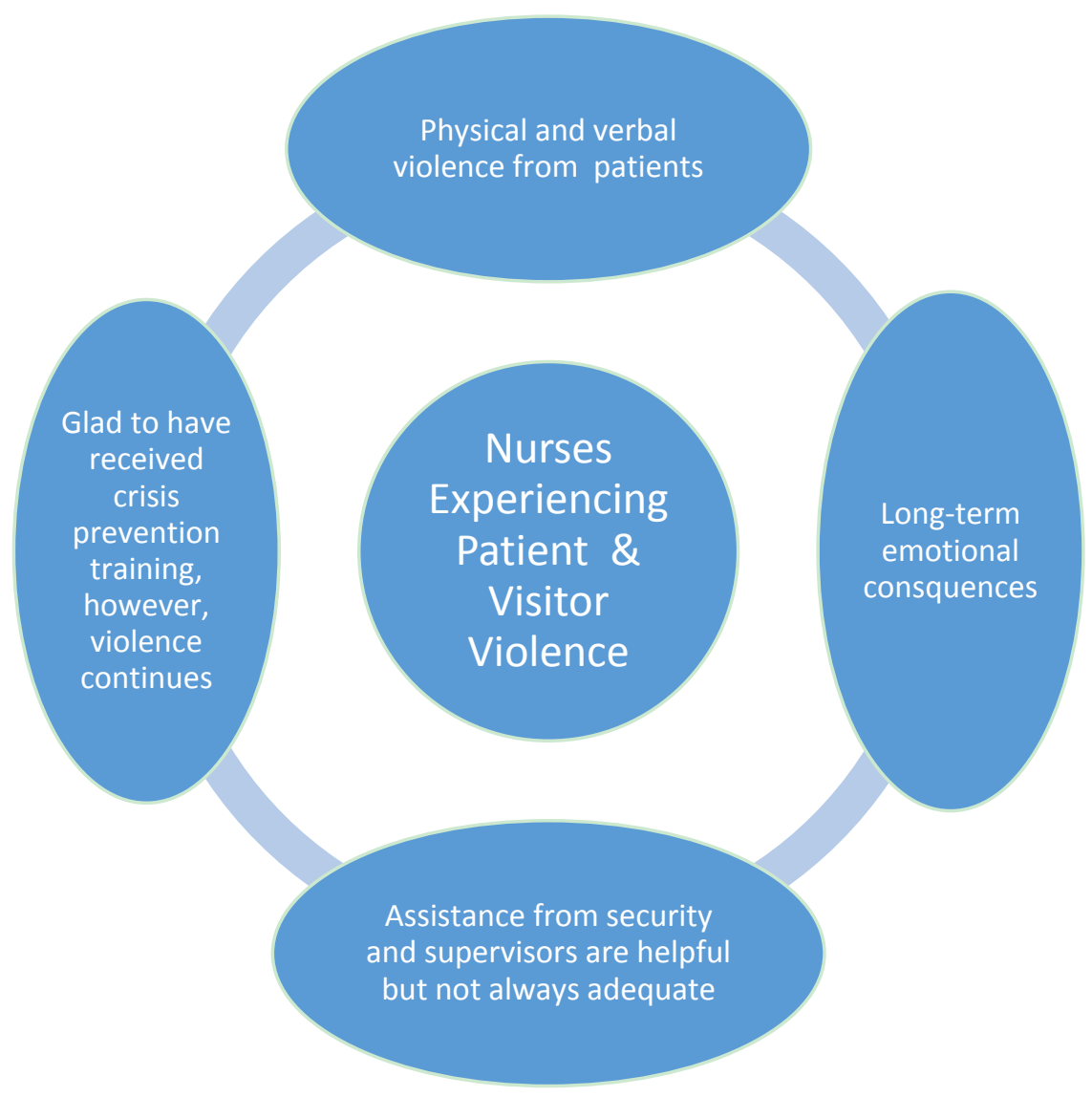

Figure 2. Common themes regarding nurses experiencing patient and visitor violence

\subsubsection{Theme 1: Violence}

The first theme that was apparent when analyzing the findings was violence. The two main types of violence that the participants stated they experienced were physical and verbal violence. The main words used by participants when related to physical violence were hitting, punching, kicking, tackling, pinching, and spitting.

Eighteen of the 19 participants stated that they had experienced some form of physical violence during their nursing career. Participant 6 (P6) stated: One time a patient grabbed my arm and dug her nails into my skin just because I was attempting to put a blood pressure cuff on her arm. This situation was really scary because she got so mad just because I was putting a blood pressure cuff on her. I did not know what to expect of her if I was going to try and do anything else for her.

Nurses experience violence from patients when they are-trying to help care for patients. P9 shared: Eventually the patient regained a full level of consciousness and tackled me to the floor. He was then placed in a therapeutic Crisis Prevention Intervention (CPI) approved hold and he was able to escape the hold. He held me on the floor with one hand and held the other back with a fist formed ready to strike me in the face. My supervisor, unit manager, and charge nurse were all present at this time. My charge nurse and supervisor were able to restrain the patient's arm that was ready to punch me.

The participants verbalized that they experienced verbal violence from patients and visitors. The participants described the verbal violence as yelling, naming calling, and swearing at the nurse. Seventeen participants expressed verbal violence from either patients or visitors. P2 stated: Just the other day I had a patient's family 
member in the hall yelling at me for not medicating the patient yet and telling me that I 'wasn't busy anyway.' Unbeknownst to this visitor, we had a full arrest in another room and I was the only RN on the floor for the rest of the patients. This patient was in the ER for a total of 45 minutes, but the visitor verbally attacked me because it took too long to get her medication and the doctor did not write for a work note. Even when I gave her a note stating she had been seen in the ED, she continued to rip it up and threaten another ED staff member in the waiting room because we were 'just being lazy and rude.'

The review of literature helps reinforce the data found. Baran-Aksakal et al. (2014) reported that $61.3 \%$ of nurses described physical violence by patient visitors and that $54.7 \%$ of the verbal abuse was from visitors as well (p. 1361). The data showed that $94.7 \%$ of the participants experienced some form of physical violence from a patient or visitor. Results also indicated that $89.5 \%$ of the participants experienced verbal violence from patients or visitors.

\subsubsection{Theme 2: Long Term Emotional Consequences of Violence}

Another theme identified was the long-term consequences of abuse on nurses. The participants expressed similar statements about how they were affected by violence. Seventeen participants expressed being more cautious or being more aware of their patients and visitors when they are caring for them. Thirteen of the participants expressed having long-term emotional affects after experiencing violence in the workplace. P3 expressed how a patient's violent outburst affected them emotionally: As I started the IV, I successfully hit a vein smoothly. I connected the IV catheter to the transfer hub to collect blood. All of a sudden, the patient flipped a switch. He yelled, "No don't f.....g draw my blood." He drew the arm I was working with back, causing the IV catheter to come out. This patient was also HIV+ and Hepatitis B+. Blood was spraying everywhere. With that same arm, the patient made a fist up in the air and attempted to swing at me. I luckily got away uninjured without a drop of his blood on myself. I have never been so scared in my life before. I was shaking.

Nurses have found themselves changing how they interact with patients and visitors following violent experiences. P7 stated: I have become more anxious at times when caring for patients because you never know what someone is going to do at any second especially if they are under the influence of some type of drug. The literature review helps reinforce the outcomes of the data analysis. Avander et al. (2016) described when violent situations occurred in the workplace; nurses were often afraid of how it would affect their lives outside of the hospital (p. 55). P1 explained best how patient violence affected her daily life: When I first became a nurse I was working at a city hospital caring for a person that was apparently the leader of a gang in the area. He took the food away from his girlfriend and proceeded to eat it. When I said something, I got a look from him. When I walked out, the "friends" in the room proceeded to follow me out in an intimidating way. The other techs said to me "do you know who you were just correcting?" I would park my car in a different spot for a while.

Patient and visitor violence is affecting nurses emotionally. This nurse had to be more cautious of her surroundings inside and outside of the hospital because of this incident she experienced. It disturbed her emotionally and psychologically for a long time.

\subsubsection{Theme 3: Education and Training for Violence Intervention}

The third theme that was found when analyzing data was education provided to nurses regarding potential violence in the workplace. Common themes by participants were crisis prevention institute (CPI) training and behavioral management training. Seventeen participants received some type of education about violence from their employer. P9 stated: Personally I have received CPI training. I have been educated in and am currently an educator of Behavioral Management Training focusing on verbal de-escalation, and have currently taken on the new role of the registered behavioral B.E.R.T. nurse in our facility.

Most of the participants received CPI training through their employer. This type of training is beneficial in helping nurses learn how to properly learn holding techniques for individuals who are portraying violent outbursts physically. This class also helps them learn how to de-escalate situations that arise verbally as well.

\subsubsection{Theme 4: Support from the Organizational Leaders}

The last theme that was discovered was support from the organization. Participants used common words such as security, help from charge nurses, or receiving no help from their supervisors. Thirteen participants described having help from security when they encountered violence from a patient or a visitor. These participants shared that they received a lot of help from their organization's security when situations arose with violent patients or visitors. P7 stated: Our security is wonderful and they are always quick to respond and have our backs. They don't get irritated even if you call them and then they aren't needed. I always back up and let security-officers handle 
these violent situations because I do not want to get hurt and they are trained on how to properly handle these situations. I have a lot of trust and respect for the security staff.

Ten participants mentioned getting help from their charge nurses when violent situations happened in the workplace. P3 stated: Supervisors stood behind me in both situations, which is what prompted the patients/visitors to be escorted out of the hospital. It feels great to have support at times like these. It helps me feel like I am not alone when these situations occur and that someone has my back. The customer isn't always right in this scenario.

Finally, six participants expressed getting no help from supervisors or the organization following a violent event. The review of the literature reinforces that nurses feel that they receive no help or support from their organization following a violent situation with a patient or visitor. Edward et al. (2014) reported similar barriers to reporting violence, including poor or no support following an incident, lack of an incident reporting system, a previous incident without action, and fear of adverse occupational consequences (p. 12). P6 stated: I have not received any help from my supervisor from this situation. They just blew it off as though nothing happened. Having no help from leadership can affect how nurses feel when these incidents occur. They feel that they are not receiving any help and that it is not important that they are being treated poorly.

\section{Limitations}

A limitation of this study were the limited number of participants who were interviewed. Another limitation was that nurses did not have time to volunteer 45-60 minutes of their time to participate in the interview. Additionally, some participants were not as open about sharing their experiences. Transferability to other settings is unknown. This study does have international relevance because violence occurs worldwide. The researchers' experience of dealing with violent patients and visitors in the healthcare setting may have affected the results.

\section{Implications}

Fundamentally, acknowledgment of violence in its many forms by healthcare leadership and their response to it is pertinent to the profession. Nurse managers must recognize the extent of emotional, psychological, physical and in many cases, physiological trauma nurses experience. Furthermore, it is important to be aware of how violence affects the quality of care, functionality, and overall perception of life by nurses. Hsieh et al. (2016) concluded that hospital managers should establish a safer working environment. Healthcare staff must work together for the benefit of all involved.

\section{Conclusion}

In summary, there is significant evidence to suggest that physical, verbal, and emotional violence is a common problem experienced by nurses in the workplace. Themes identified were violence, long-term consequences of violence, education for violence prevention, and the need for support from the organization. Potential responses include education and training for healthcare staff regarding violence prevention which includes care, welfare, safety, and security of all individuals. Some violence is inevitable; however, it can be controlled and handled efficiently with sufficient staffing, supervisory support, trust, and training.

\section{Recommendations}

Violence is a rising factor in healthcare from patients and visitors toward nurses today. The first recommendation is that crisis prevention training and de-escalation education should be provided to all nurses regardless of the unit that they are working. CPI provides safe holding techniques and de-escalation techniques that can help improve the safety of dealing with violent patients and visitors. The second recommendation is that there should be protocols and policies implemented through management about follow up after a violent incidence. Many nurses do not have to follow up protocols or procedures to help nurses debrief about the situation. Nurses feel that there should be some type of follow up with their management after these incidents and support them. These recommendations are suggested after analyzing the research data. More research should be conducted, which may reinforce the validity of this study, as this is a growing problem in healthcare. It is important to note that nurses are facing violence in the workplace and this behavior is unacceptable and should be addressed. The need for further research on this topic is critical.

\section{References}

American Nurses Association. (2015). Position Statement on: Incivility, bullying and workplace violence. Retrieved from http://www.nursingworld.org/DocumentVault/Position-Statements/Practice/Position-Statement-on-Incivilit y-Bullying-and-Workplace-Violence.pdf 
Avander, K., Heikki, A., Bjersa, K., \& Engstrom, M. (2016). Trauma nurses' experience of workplace violence and threats: Short- and long-term consequences in a Swedish setting. Journal of Trauma Nursing, 23(2), 51-57. https://doi.org/10.1097/JTN.0000000000000186

Baby, M., Glue, P., \& Carlyle, D. (2014). Violence is not part of our job: A thematic analysis of psychiatric mental health nurses' experiences of patient assaults from a New Zealand perspective. Issues in Mental Health Nursing, 35(9), 647-655. https://doi.org/10.3109/01612840.2014.892552

Baran-Aksakal, F.N., Karasahin, E.F., Urgas-Dikmen, A., Avci, E., \& Ozkan, S. (2014). Workplace physical violence, verbal violence and mobbing experienced by nurses at a university hospital. Turkish Journal of Medical Sciences, 45, 1360-1368. https://doi.org/10.3906/sag-1405-65

Bureau of Labor Statistics. (2016). Non-fatal occupational injuries and illnesses requiring days away from work, 2015. United States Department of Labor. Retrieved from www.http://1.usa.gov/1LlwcDX

Edward, K., Ousey, K., Warelow, P., \& Lui, S. (2014). Nursing and aggression in the workplace: A systemic review. British Journal of Nursing, 23(12), 653-659. https://doi.org/10.12968/bjon.2014.23.12.653

Hsieh, H.F., Chen, Y.M., Wang, H.H., Chang, S.C, \& Ma, S.C. (2016). Association among components of resilience and workplace violence-related depression among emergency department nurses in Taiwan: A cross-sectional study. Journal of Clinical Nursing, 25, 2639-2647. https://doi.org/10.1111/jcon.13309

Kvas, A., \& Seljak, J. (2014). Unreported workplace violence in nursing. International Nursing Review, 61(3), 344-351. https://doi.org/10.1111/inr.12106

Occupational Safety and Health Administration. (2015). A workplace violence. Retrieved from http://nursingworld.org/workplaceviolence

Sanders, C. (2003). Application of Colaizzi's method: Interpretation of an auditable decision trail by a novice researcher. Contemporary Nurses Journal, 14(3), 292-302. Retrieved from https//www.ncbi.nlm.nih.gov/pubmed/12868668

Schmidt, N.A., \& Brown, J.M. (2015). Evidence-based practice for nurses: Appraisal and application of research. Burlington, MA: Jones \& Bartlett Learning.

Stene, J., Larson, E., Levy, M., \& Dohlman, M. (2015). Workplace violence in the emergency department: Giving staff the tools and support to report. The Permanente Journal, 19(2), 113-117. http://doi.org/10.7812/TPP/14-187

Stevenson, K.N., Jack, S.M., O’Mara, L., \& LeGris, J. (2015). Registered nurses' experiences of patient violence on acute care psychiatric inpatient units: An interpretive descriptive study. BMC Nursing, 14(35), 1-13. https://doi.org/10.1186/s12912-015-0079-5

Taylor, S.E. (1983). Adjustments to threatening events: A theory of cognitive adaptation. American Psychologist, 38(11), 1161-1173. Retrieved from http://66.199.228.237/boundary/Childhood_trauma_and_PTSD/adjustment_to_threating_events.pdf

Trahan, R.L., \& Bishop, S.L. (2016). Coping strategies of neurology nurses experiencing abuse from patients and families. Journal of Neuroscience Nursing, 48(3), 118-123. https://doi.org/10.1097/JNN.0000000000000211

World Health Organization. (2017). Retrieved http://www.who.int/violence_injury_prevention/violence/workplace/en/

Zhao, S., Liu, H., Ma, H., Jiao, M., Li, Y., Hao, Y., ... Qiao, H. (2015). Coping with Workplace Violence in Healthcare Settings: Social Support and Strategies. International Journal of Environmental Research and Public Health, 12(11), 14429-14444. https://doi.org/10.3390/ijerph121114429

\section{Copyrights}

Copyright for this article is retained by the author(s), with first publication rights granted to the journal.

This is an open-access article distributed under the terms and conditions of the Creative Commons Attribution license (http://creativecommons.org/licenses/by/4.0/). 